\title{
BMJ Open Association between biomarkers and COVID-19 severity and mortality: a nationwide Danish cohort study
}

Gethin Hodges (D) , ${ }^{1}$ Jannik Pallisgaard, ${ }^{1}$ Anne-Marie Schjerning Olsen, ${ }^{2,3}$ Patricia McGettigan, ${ }^{4}$ Mikkel Andersen, ${ }^{5}$ Maria Krogager, ${ }^{5,6}$ Kristian Kragholm, ${ }^{6}$ Lars Køber, ${ }^{7}$ Gunnar Hilmar Gislason, ${ }^{1,3,8}$ Christian Torp-Pedersen,, 6 Casper N Bang ${ }^{3,7,10}$

To cite: Hodges G, Pallisgaard J, Schjerning Olsen A-M, et al. Association between biomarkers and COVID-19 severity and mortality: a nationwide Danish cohort study. BMJ Open 2020;10:e041295. doi:10.1136/ bmjopen-2020-041295

- Prepublication history and additional material for this paper are available online. To view these files, please visit the journal online (http://dx.doi org/10.1136/bmjopen-2020041295).

Received 06 June 2020 Revised 23 September 2020 Accepted 11 November 2020

D Check for updates

(c) Author(s) (or their employer(s)) 2020. Re-use permitted under CC BY-NC. No commercial re-use. See rights and permissions. Published by BMJ.

For numbered affiliations see end of article.

\section{Correspondence to}

Dr Gethin Hodges;

gethin.william.hodges@regionh. $\mathrm{dk}$

\section{ABSTRACT}

Objective To evaluate the association between common biomarkers, death and intensive care unit (ICU) admission in patients with COVID-19.

Design Retrospective cohort study. From electronic national registry data, we used Cox analysis and bootstrapping to evaluate associations between baseline levels of biomarkers and standardised absolute risks of death/ICU admission, adjusted for age and gender.

Setting All hospitals in Denmark.

Participants 1310 patients aged $\geq 18$ years admitted to hospital with COVID-19 from 27th of February to 1st of May 2020, with available biochemistry data.

Main outcome measures A composite of death/ICU admission occurring within 30 days.

Results of the 1310 patients admitted to hospital (54.6\% men; median age 73.6 years), 352 (26.9\%) experienced the composite endpoint and $263(20.1 \%)$ died. For the composite endpoint, the absolute risks for moderately and severely elevated $\mathrm{C}$ reactive protein (CRP) were significantly higher, $21.5 \%$ and $39.2 \%$, respectively, compared with $5.0 \%$ for those with normal CRP. Moderately and severely elevated leucocytes were significantly higher, $34.5 \%$ and $46.6 \%$ risk, respectively, compared with $23.2 \%$ for those with normal leucocytes. Moderately and severely decreased estimated glomerular filtration rates (eGFR) were significantly higher, $41.5 \%$ and $45.9 \%$ risk, respectively, compared with $30.4 \%$ for those with normal/mildly decreased eGFR. Normal and elevated ureas were significantly higher, $22.3 \%$ and $40.6 \%$ risk, respectively, compared with $7.3 \%$ for those with low urea. Elevated D-dimer was significantly higher, $31.8 \%$ risk, compared with $17.5 \%$ for those with normal D-dimer. Moderately and severely elevated troponins were significantly higher, $27.7 \%$ and $57.3 \%$ risk, respectively, compared with $9.4 \%$ for those with normal troponin. Elevated procalcitonin was significantly higher, $52.1 \%$ risk, compared with $28.0 \%$ for those with normal procalcitonin.

Conclusion In this nationwide study of patients admitted with COVID-19, elevated levels of CRP, leucocytes, procalcitonin, urea, troponins and D-dimer, and low levels of eGFR were associated with higher standardised absolute risk of death/ICU admission within 30 days.
Strengths and limitations of this study

- Much of the research concerning COVID-19 describes small case studies from China and Italy, without clearly defined outcomes.

- This study is the first to report the standardised absolute risk of individual laboratory tests on short-term mortality and ICU admission in a relatively large, European cohort of 1310 patients with COVID-19.

- Our study can help the clinicians to understand which biomarkers are important in identifying patients with poor prognosis, which may be useful to assess disease severity or to enable early intervention.

- The main limitation of our study is its observational, non-randomised design.

- This study included only patients admitted to the hospital with COVID-19 and measured biochemical data, hence it is likely to represent symptomatic patients at the more severe end of the disease spectrum.

\section{INTRODUCTION}

COVID-19 caused by the novel coronavirus, SARS-CoV-2, has spread rapidly to become a worldwide pandemic resulting in an enormous strain on healthcare systems globally. As of 20th of September 2020, the number of confirmed cases has surpassed 30 million, affecting 213 countries, although the actual number is likely to be much higher. ${ }^{1}$ The clinical course of COVID-19 is variable, but is typically characterised by an initial phase with fever or mild upper respiratory symptoms (though many are asymptomatic). Among hospitalised patients, those with a poor prognosis tend to develop severe viral pneumonia requiring ventilatory support and intensive care unit (ICU) admission. ${ }^{2}$ Despite supportive care, a high proportion of patients with COVID-19 suffer rapid deterioration with respiratory failure and death. ${ }^{3-5}$ Identifying which patients are at risk of severe disease or 
death, may be useful in decision-making, to determine whether hospitalisation or ICU referral is required or to enable early intervention. ${ }^{6}$ This is of particular importance given that the fast pace of the pandemic has led to rationing of scarce resources, most notably mechanical ventilators. ${ }^{7}$

Several studies have demonstrated that older age and chronic diseases are associated with poor outcome in patients with COVID-19. ${ }^{25}$ Furthermore, in a recently published systematic review, disease severity was associated with more prominent laboratory abnormalities including markers of inflammation and organ damage including elevated troponins, although much of the early research describes small case studies without clearly defined outcomes and the need for further research in more varied cohorts was highlighted. ${ }^{28-11} \mathrm{~A}$ recently published study showed that in multivariate analysis, older age, higher Sequential Organ Failure Assessment (SOFA) score and elevated D-dimer on admission were independently associated with in-hospital death. ${ }^{5}$ The present study aims to expand on these findings and evaluate which biomarkers are associated with death and ICU admission in a large nationwide cohort.

\section{METHODS}

\section{Data sources}

This study is based on four nationwide registers: the Danish National Patient Register, the Civil Registration System, the Danish Registry of Medicinal Product Statistics and the database on blood samples (LABKA). These four nationwide registers were cross-linked on the individual level using the unique permanent identification number given to all Danish residents at birth or migration.

The Danish National Patient Register holds information on every hospital visit in Denmark, in which each visit is registered with a diagnosis according to the International Classification of Diseases, the 10th revision. The Civil Registration System holds information on the day of birth, sex and vital status. The Danish Registry of Medicinal Product Statistics contains information on all prescriptions dispensed from Danish pharmacies and is coded according to the Anatomical Therapeutic Chemical classification system. ${ }^{12}$ The LABKA database holds information on blood samples from all hospital visits, including the emergency departments, outpatient consultations and admissions to the hospital.

\section{Study design and participants}

This study included all patients with laboratory-confirmed COVID-19 aged 18 years and older with available biochemistry data, admitted to hospital between 27th of February 2020 and 1st of May 2020 (the first Danish case was recorded on 27th of February 2020). Patients were included on the first day of diagnosis with COVID-19 and followed for 30 days for the combined endpoint of all-cause mortality and ICU admission. A subanalysis was also performed for the endpoint of all-cause mortality alone. Blood test results were obtained from electronic registries of laboratory data, with baseline values taken on admission (measured within 24 hours). We focused on readily available laboratory tests associated with inflammation or organ damage, including $\mathrm{C}$ reactive protein (CRP), ferritin, procalcitonin, leucocyte count, estimated glomerular filtration rate (eGFR), urea, alanine aminotransferase (ALAT), D-dimer and troponin (both T and I). The eGFR was calculated using the Modification of Diet in Renal Disease equation, which includes creatinine level, age, race and sex. In order to compare troponin values with different assays and reference values, a ratio between observed values and highest reference values was performed.

\section{Statistical analysis}

Categorical data were presented as counts with percentages, and the statistical difference was tested using Fisher's exact test. Continuous variables were presented as medians with the first and third quartile $(\mathrm{Q} 1$ and Q3), and the statistical difference was tested using the Wilcoxon rank-sum test. Cox analysis and bootstrapping with 100 bootstraps were used to derive age-adjusted and gender-adjusted standardised absolute risk and average treatment effects curves with $95 \%$ CIs to evaluate the association between individual biomarkers and the 30-day risk for each endpoint. The above analysis was repeated with stratification by typical normal/elevated ranges used in the clinical setting. For the stratified analysis of troponin, the cut-off values were defined as being moderately elevated (>one to two times elevated) and severely elevated (>two times elevated), relative to baseline troponin. As part of a sensitivity analysis, we performed a Cox multivariate regression analysis to assess CRP in relation to the combined endpoint of all-cause mortality and ICU admission (adjusted for age, gender, diabetes, chronic obstructive pulmonary disease (COPD), hypertension and ischaemic heart disease). A two-sided $p$ value of $\leq 0.05$ was considered statistically significant.

Data management and statistical analyses were conducted using R statistics (R Core Team (2020); R: a language and environment for statistical computing, $\mathrm{R}$ Foundation for Statistical Computing, Vienna, Austria; https://www.R-project.org/).

\section{RESULTS}

A total of 4444 patients with COVID-19 were identified in the study period. We excluded 28 patients aged below 18 years old, 2653 patients who were not admitted to hospital and 453 with no available biochemistry data, leaving 1310 patients for inclusion in the study $(54.6 \%$ men; median age 73.6 years). Of these, $352(26.9 \%)$ patients experienced the composite endpoint and 263 (20.1\%) died (see figure 1).

Baseline characteristics for the total cohort and stratified by the composite endpoint are given in table 1 (see also online supplemental table S1 for baseline characteristics 


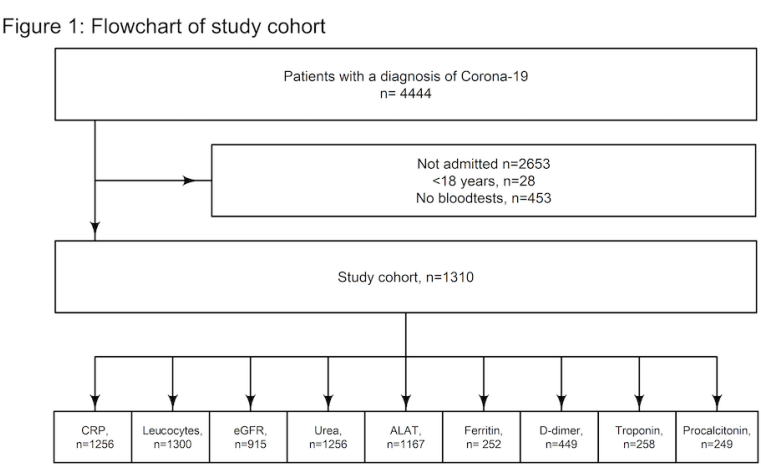

Legend: ALAT, alanintransaminase; CRP, C-reactive protein; eGFR, estimated glomerular filtration rate

Figure 1 Flowchart of study cohort. ALAT, alanine aminotransferase; CRP, C reactive protein; eGFR, estimated glomerular filtration rate.

stratified by death and online supplemental table S2 stratified according to availability of biochemistry). Patients who experienced the composite endpoint of death/ ICU admission within 30 days were more likely to be older, men, with a pre-existing comorbidity (diabetes, COPD, atrial fibrillation, hypertension or heart failure); currently receiving treatment with aspirin, beta-blocker, angiotensin II receptor blockers, loop diuretics, calcium channel blockers or spironolactone; with higher baseline values of leucocytes, urea, D-dimer, troponin or procalcitonin; or lower baseline values of eGFR ( $\mathrm{p} \leq 0.044$ for all). However, the proportion of patients who died or were admitted to ICU was not significantly different for those with prior ischaemic stroke, ischaemic heart disease, chronic kidney disease, cancer, or currently receiving treatment with non-steroidal anti-inflammatory drugs or thiazides.

\section{Biomarkers and standardised absolute risk of death and ICU admission}

$\mathrm{C}$ reactive protein

Higher baseline CRP was associated with higher ageadjusted and sex-adjusted absolute risk of death/ICU admission (figure 2), and death alone (online supplemental figure S1).

In stratified analysis, moderately elevated (upper reference limit to $99 \mathrm{mmol} / \mathrm{L}$ ) and severely elevated (100-400 mmol/L) baseline CRP were associated with an age-adjusted and sex-adjusted absolute risk of $21.5 \%$ (95\% CI: $18.1 \%$ to $24.9 \%$ ) and $39.2 \%$ (95\% CI: $35.6 \%$ to $43.0 \%$ ) for death/ICU admission within 30 days, respectively, which was a significantly higher risk (both $\mathrm{p}<0.001$ ) compared with those with normal CRP, absolute risk $5.0 \%$ (95\% CI: $0.0 \%$ to $12.0 \%$ ) (figure 3 ).

Similarly, moderately and severely elevated baseline CRP were associated with an age-adjusted and sexadjusted absolute risk of $18.1 \%$ (95\% CI: $15.1 \%$ to $21.3 \%$ ) and $28.8 \%$ (95\% CI: $25.4 \%$ to $32.1 \%$ ) for 30-day mortality, respectively, which was a significantly higher risk (both $\mathrm{p}<0.001)$ compared with those with normal CRP, absolute risk $6.6 \%$ (95\% CI: $0.0 \%$ to $15.0 \%$ ) (online supplemental figure $\mathrm{S} 2)$.
In a multivariate model, elevated CRP was independently associated with death/ICU admission after adjusting for age, gender, diabetes, COPD, hypertension and ischaemic heart disease (figure 4; online supplemental table S3).

\section{Leucocytes}

Higher baseline leucocyte count was associated with higher age-adjusted and sex-adjusted absolute risk of death/ICU admission (figure 2), and death alone (online supplemental figure $\mathrm{S} 1$ ).

In stratified analysis, moderately elevated (upper reference limit to $15 \times 10^{9} / \mathrm{L}$ ) and severely elevated $\left(15-30 \times 10^{9} / \mathrm{L}\right)$ baseline leucocytes were associated with an age-adjusted and sex-adjusted absolute risk of $34.5 \%$ (95\% CI: $29.5 \%$ to $39.4 \%$ ) and $46.6 \%$ (95\% CI: $38.5 \%$ to $54.6 \%$ ) for death/ICU admission within 30 days, respectively, which was a significantly higher risk (both $\mathrm{p}<0.001$ ) compared with those with normal leucocytes, absolute risk $23.2 \%$ (95\% CI: $20.4 \%$ to $22.2 \%$ ) (figure 3 ).

In stratified analysis, moderately and severely elevated baseline leucocytes were associated with an age-adjusted and sex-adjusted absolute risk of $26.6 \%$ (95\% CI: $22.0 \%$ to $31.3 \%$ ) and $37.0 \%$ (95\% CI: $25.4 \%$ to $32.1 \%$ ) for 30 -day mortality, respectively, which was a significantly higher risk (both $\mathrm{p}<0.001$ ) compared with those with normal leucocytes, absolute risk $17.6 \%$ (95\% CI: $15.0 \%$ to $20.2 \%$ ) (online supplemental figure S2).

\section{Estimated glomerular filtration rate}

Lower baseline eGFR was associated with higher ageadjusted and sex-adjusted absolute risk of death/ICU admission (figure 2), and death alone (online supplemental figure $\mathrm{S} 1$ ).

In stratified analysis, moderately decreased (30-60 mmol/L) and severely decreased (0-30 mmol/L) baseline eGFR were associated with an age-adjusted and sex-adjusted risk of $41.5 \%$ (95\% CI: $35.1 \%$ to $48.0 \%$ ) and $45.9 \%$ (95\% CI: $34.9 \%$ to $56.8 \%$ ) for death/ICU admission within 30 days, respectively, which was a significantly higher risk (both $\mathrm{p}<0.001$ ) compared with those with normal/mild decreased eGFR ( $>60 \mathrm{mmol} / \mathrm{L})$, absolute risk $30.4 \%$ (95\% CI: $26.7 \%$ to $34.1 \%$ ) (figure 3 ).

In stratified analysis, moderately elevated and severely elevated baseline eGFR were associated with an absolute risk of $34.8 \%$ (95\% CI: $29.0 \%$ to $40.5 \%$ ) and $42.4 \%$ (95\% CI: $32.9 \%$ to $52.0 \%$ ) for 30-day mortality, respectively, (figure 3), which was a significantly higher risk (both $\mathrm{p}<0.001$ ) compared with those with normal $/ \mathrm{mild}$ decreased eGFR, absolute risk $22.0 \%$ (95\% CI: $18.5 \%$ to $25.5 \%$ ) (online supplemental figure S2).

\section{Urea}

Higher baseline urea was associated with higher ageadjusted and sex-adjusted absolute risk of death/ICU admission (figure 2), and death alone (online supplemental figure $\mathrm{S} 1$ ). 
Table 1 Characteristics of patients with COVID-19 for total cohort, and stratified by death/ICU admission (within 30 days of diagnosis)

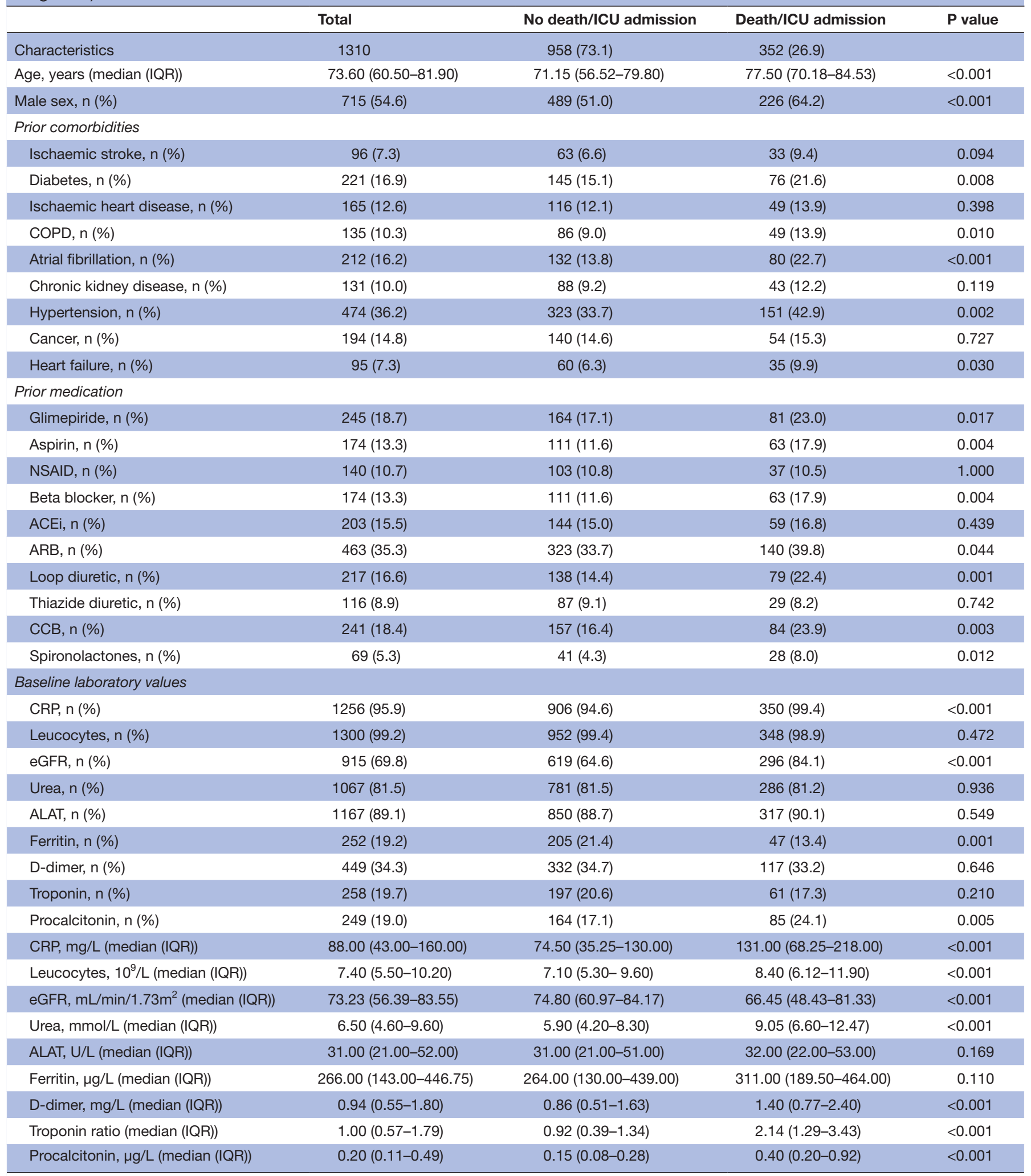

ACEi, ACE inhibitor; ALAT, alanine aminotransferase; ARB, angiotensin receptor blocker; CCB, calcium channel blocker; COPD, chronic obstructive pulmonary disease; CRP, C reactive protein; eGFR, estimated glomerular filtration rate; ICU, intensive care unit; NSAID, non-steroidal anti-inflammatory drug.

In stratified analysis, normal $(3.6-8.0 \mathrm{mmol} / \mathrm{L})$ and elevated $(>8 \mathrm{mmol} / \mathrm{L})$ baseline ureas were associated with an absolute risk of $22.3 \%$ (95\% CI: $18.7 \%$ to $25.9 \%$ ) and $40.6 \%$ (95\% CI: $35.5 \%$ to $45.7 \%$ ) for death/
ICU admission within 30 days, respectively, which was a significantly higher risk (both $\mathrm{p}<0.001$ ) compared with those with low urea, absolute risk $7.3 \%$ (95\% CI: $1.6 \%$ to $12.9 \%$ ) (figure 3 ). 
Figure 2: 30-day absolute risk for the composite outcome of death or ICU admission, adjusted for age and
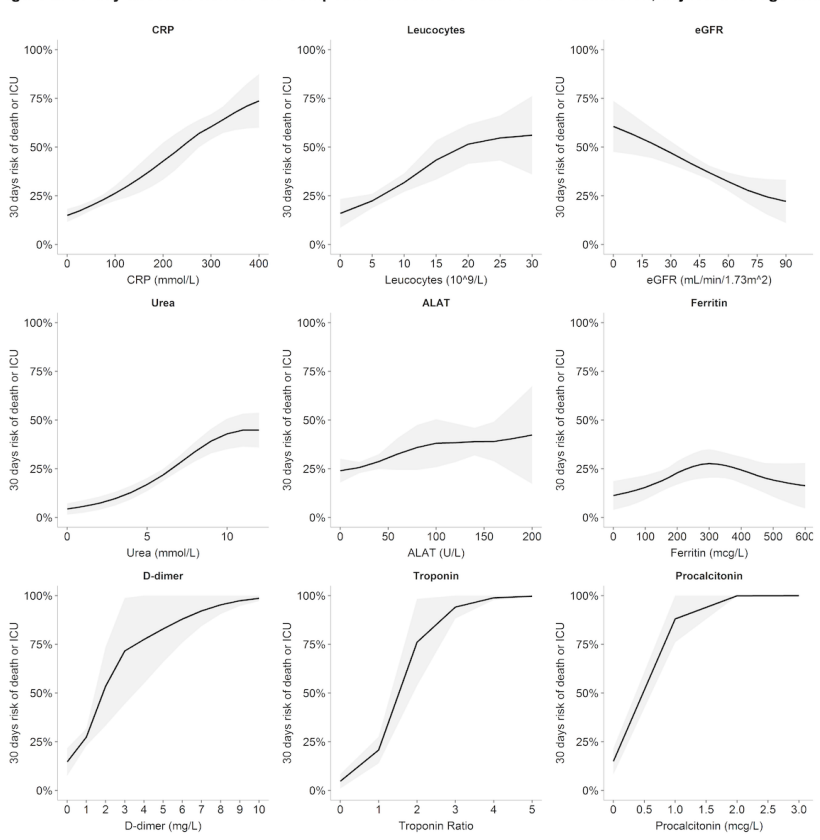

Legend: ALAT, alanintransaminase; CRP, C-reactive protein; eGFR, estimated glomerular filtration rate; ICU, intensive care unit

Figure 2 A 30-day absolute risk for the composite outcome of death or ICU admission, adjusted for age and gender. ALAT, alanine aminotransferase; CRP, $C$ reactive protein; eGFR, estimated glomerular filtration rate; ICU, intensive care unit.

In stratified analysis, normal and elevated baseline ureas were associated with an absolute risk of $15.7 \%$ (95\% CI: $11.2 \%$ to $17.4 \%$ ) and $31.9 \%$ (95\% CI: $26.8 \%$ to $35.4 \%$ )

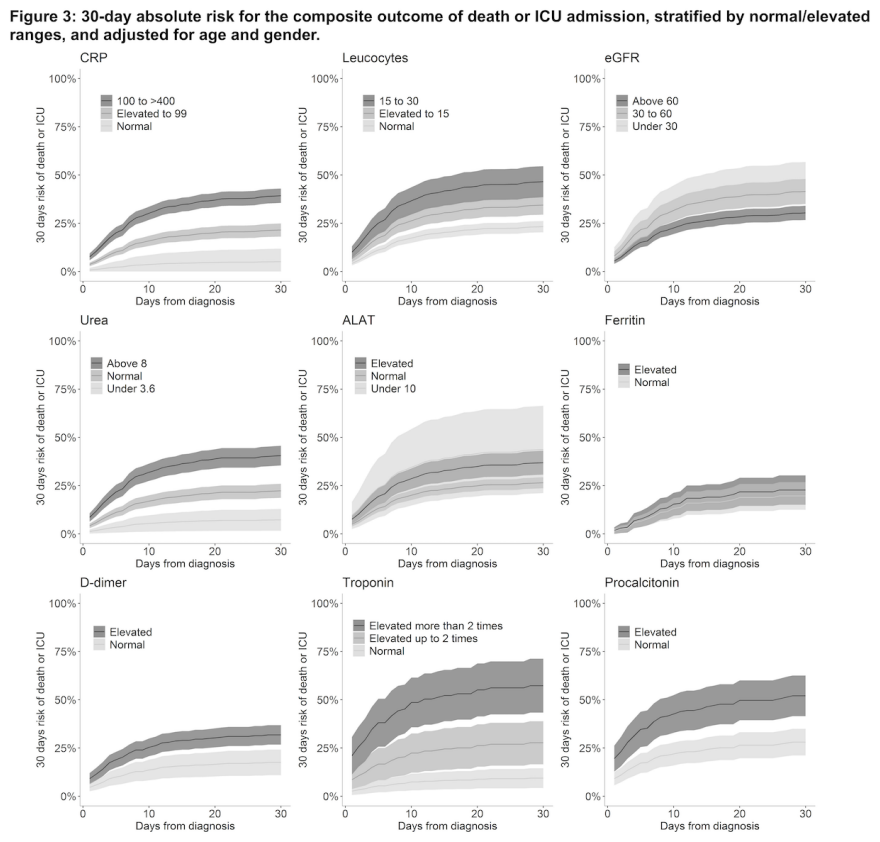

Legend: ALAT, alanintransaminase; CRP. C-reactive protein: EGFR, estimated glomerular filtration rate: ICU, intensive care unit.

Figure 3 A 30-day absolute risk for the composite outcome of death or ICU admission, stratified by normal/elevated ranges, and adjusted for age and gender. ALAT, alanine aminotransferase; CRP, $\mathrm{C}$ reactive protein; eGFR, estimated glomerular filtration rate; ICU, intensive care unit.

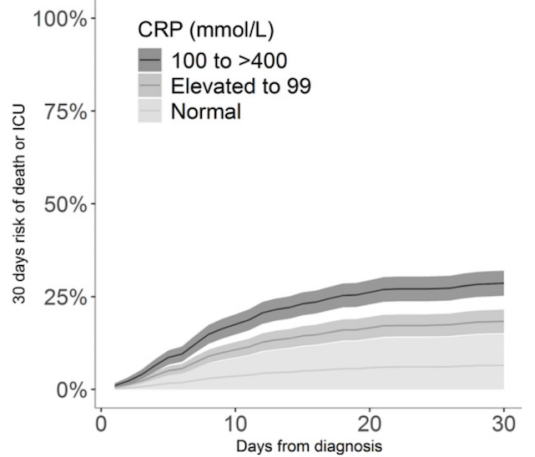

Figure 4 A 30-day absolute risk for the composite outcome of death or ICU admission, adjusted for CRP level, age, gender, diabetes, chronic obstructive pulmonary disease, hypertension and ischaemic heart disease. CRP, $\mathrm{C}$ reactive protein; ICU, intensive care unit.

for 30-day mortality, respectively, which was a significantly higher risk (both $\mathrm{p}<0.001$ ) compared with those with low urea, absolute risk $5.4 \%$ (95\% CI: $0.0 \%$ to $11.0 \%$ ) (online supplemental figure $\mathrm{S} 2$ ).

\section{Alanine aminotransferase}

Higher baseline ALAT was associated with slightly higher absolute risk of death/ICU admission (figure 2), and death alone (online supplemental figure S1).

In stratified analysis, elevated (>upper reference limit) baseline ALAT was associated with an absolute risk of $36.9 \%$ (95\% CI: $30.7 \%$ to $43.1 \%$ ) for death/ICU admission within 30 days, which was significantly higher than normal ALAT ( $10 \mathrm{U} / \mathrm{L}$ to upper reference limit), absolute risk $26.9 \%$ (95\% CI: $23.6 \%$ to $29.3 \%$, p=0.002), however not significantly different to low ALAT $(<10 \mathrm{U} / \mathrm{L})$, absolute risk $43.8 \%$ (95\% CI: $21.1 \%$ to $66.4 \%$ ) (figure 3).

In stratified analysis, low baseline ALAT was associated with an absolute risk of $41.0 \%$ (95\% CI: $22.7 \%$ to $59.4 \%$ ) for 30-day mortality, which was significantly higher than normal ALAT, absolute risk 20.8\% (95\% CI: $18.2 \%$ to $23.4 \%, \mathrm{p}=0.03$ ), however not significantly different to high ALAT, absolute risk $25.8 \%$ (95\% CI: $19.9 \%$ to $31.8 \%$ ) (online supplemental figure S2).

Ferritin

Higher baseline ferritin was associated with an inverted $\mathrm{U}$ shaped risk of death/ICU admission (figure 2), and risk of death alone (online supplemental figure S1).

In stratified analysis, elevated $(>300 \mathrm{mmol} / \mathrm{L})$ baseline ferritin was associated with an age-adjusted and sexadjusted absolute risk of $22.7 \%$ (95\% CI: $15.1 \%$ to $30.3 \%$ ) for death/ICU admission within 30 days, which was not a significantly higher risk compared with those with normal ferritin $(\leq 300 \mathrm{mmol} / \mathrm{L})$, absolute risk $19.7 \%$ (95\% CI: $12.5 \%$ to $32.9 \%$ ) (figure 3 ).

In stratified analysis, elevated $(>300 \mathrm{mmol} / \mathrm{L})$ baseline ferritin was associated with an age-adjusted and sexadjusted risk of $15.9 \%$ (95\% CI: $0.09 \%$ to $22.8 \%$ ) for 30-day mortality, which was not a significantly higher risk compared with those with normal ferritin $(\leq 300 \mathrm{mmol} / \mathrm{L})$, 
absolute risk $19.8 \%$ (95\% CI: $12.6 \%$ to $26.9 \%$ ) (online supplemental figure S2).

\section{D-dimer}

Higher baseline D-dimer was associated with an ageadjusted and sex-adjusted higher risk of death/ICU admission (figure 2), and risk of death alone (online supplemental figure $\mathrm{S} 1$ ).

In stratified analysis, elevated $(>0.5 \mathrm{mg} / \mathrm{L})$ baseline D-dimer was associated with an age-adjusted and sexadjusted absolute risk of $31.8 \%$ (95\% CI: $26.7 \%$ to $36.8 \%$ ) for death/ICU admission within 30 days, which was a significantly higher risk $(\mathrm{p}<0.001)$ compared with those with normal D-dimer, absolute risk $17.5 \%$ (95\% CI: $10.9 \%$ to $24.1 \%$ ) (figure 3 ).

In stratified analysis, elevated baseline D-dimer was associated with an age-adjusted and sex-adjusted risk of $19.1 \%$ (95\% CI: $14.7 \%$ to $23.5 \%$ ) for 30-day mortality, which was a significantly higher risk $(\mathrm{p}<0.001)$ compared with those with normal D-dimer, absolute risk 13.5\% (95\% CI: 8.5\% to $19.5 \%$ ) (online supplemental figure S2).

\section{Troponin}

Higher baseline troponin ratio was associated with ageadjusted and sex-adjusted higher risk of death/ICU admission (figure 2), and risk of death alone (online supplemental figure S1).

In stratified analysis, moderately elevated and severely elevated baseline troponins were associated with an ageadjusted and sex-adjusted absolute risk of $27.7 \%$ (95\% CI: $16.5 \%$ to $38.9 \%$ ) and $57.3 \%$ (95\% CI: $43.3 \%$ to $71.3 \%$ ) for death/ICU admission within 30 days, respectively, which was a significantly higher absolute risk $(\mathrm{p}=0.003$ and $\mathrm{p}<0.001$, respectively) compared with those with normal troponins, absolute risk $9.4 \%$ (95\% CI: $4.2 \%$ to $14.5 \%$ ) (figure 3).

In stratified analysis, moderately elevated and severely elevated baseline troponins were associated with an ageadjusted and sex-adjusted absolute risk of $10.8 \%$ (95\% CI: $3.2 \%$ to $18.5 \%$ ) and $35.9 \%$ (95\% CI: $23.2 \%$ to $48.6 \%$ ) for 30-day mortality, respectively. However, only the latter was a significantly higher risk $(\mathrm{p}<0.001)$ compared with those with normal troponins, absolute risk 3.9\% (95\% CI: $0.0 \%$ to $8.7 \%$ ) (online supplemental figure S2).

\section{Procalcitonin}

Higher baseline procalcitonin was associated with ageadjusted and sex-adjusted higher risk of death/ICU admission (figure 2), and risk of death alone (online supplemental figure $\mathrm{S} 1$ ).

In stratified analysis, elevated $(>0.5 \mu \mathrm{g} / \mathrm{L})$ baseline procalcitonin was associated with an age-adjusted and sex-adjusted risk of $52.1 \%$ (95\% CI: $41.5 \%$ to $62.6 \%$ ) for death/ICU admission within 30 days, which was a significantly higher risk $(\mathrm{p}<0.001)$ compared with those with normal procalcitonin, absolute risk $28.0 \%$ (95\% CI: $21.1 \%$ to $34.9 \%$ ) (figure 3 ).
In stratified analysis, elevated baseline procalcitonin was associated with an age-adjusted and sex-adjusted absolute risk of $29.5 \%$ (95\% CI: $19.9 \%$ to $39.0 \%$ ) for 30 -day mortality, which was a significantly higher absolute risk $(\mathrm{p}=0.03)$ compared with those with normal procalcitonin, absolute risk $18.0 \%$ (95\% CI: $12.0 \%$ to $24.0 \%$ ) (online supplemental figure $\mathrm{S} 2$ ).

\section{DISCUSSION}

This nationwide study is the first of its kind to examine the association between common biomarkers and risk of early death and ICU admission in adult patients admitted to hospital with laboratory-confirmed COVID19. In particular, the inflammatory markers CRP, leucocytes and procalcitonin, and markers of organ damage including eGFR, troponins and D-dimer are associated with higher risk of death/ICU admission within 30 days. However, the association between ferritin and ALAT was non-significant.

The novelty of the SARS-CoV-2, coupled with the rapid spread of the COVID-19 pandemic, has led to a tremendous burden on healthcare systems worldwide. To provide optimal care for patients, early diagnosis and identification of vulnerable patients who are at risk of severe disease is needed, as well as recognising patients who may rapidly deteriorate and require ICU admission and mechanical ventilation. This relies on an accurate knowledge of the critical clinical predictors for disease progression in order to triage patients and allocate scarce resources efficiently. This is especially important when considering which patients should start treatment. For example, in the first trial to offer an effective treatment for COVID-19 disease, results showed that early administration of antiviral treatment with remdesivir was superior to placebo in reducing time to recovery and in particular, the authors highlighted the need to start antiviral treatment before pulmonary disease progresses to require mechanical ventilation. ${ }^{13}$

In the present study, patients who died or were admitted to ICU were more likely to be older, men and with a preexisting comorbidity, which is consistent with observed global trends. Our results are aligned with a recent large cohorts of patients hospitalised with COVID-19 in the UK and USA, as well as meta-analysis of studies from China. ${ }^{2} 14$ The median age of patients who died in our study was 81 years, similar to the values noted in the UK study (median 80 years) but much older compared with patients included in the early studies from the Wuhan region. Similarly, we reported a greater proportion of death/ICU admission among men $(64 \%)$ and patients with pre-existing comorbidity (prior diabetes, COPD, atrial fibrillation and hypertension). Differences are most likely due to a variety of factors including regional differences concerning demographics, preparedness and knowledge of COVID-19 that have drawn on experience from China and other countries as the outbreak has spread. 
In terms of clinical laboratory biomarkers, recent systematic reviews of the literature have highlighted many of the challenges inherent in the early studies of COVID19 , as many of the early studies are limited by the small size of the study populations with regional bias (most data are confined to China and Italy), and with limited or poorly defined outcomes. ${ }^{611}$ Several studies have evaluated level of individual biomarkers in small and selected COVID-19 cohorts. An Italian study, including 239 patients, reported that several laboratory parameters including elevated lymphocytes, procalcitonin, interleukin- 6 , ferritin, CRP and ALAT were associated with death and ICU admission in unadjusted Cox analysis. However, in adjusted analysis, CRP was the only biomarker associated with increased risk of death/ICU admission. ${ }^{15}$

An early meta-analysis identified a range of abnormal biomarkers that was elevated in patients with COVID-19 who died, including elevated levels of inflammatory markers and acute phase reactants such as interleukin-6, CRP, ferritin, lymphopenia, as well as reduced CD4 and CD8 counts and coagulation abnormalities including prolonged PT, increased D-dimer and thrombocytopenia. ${ }^{16}$ We were able to expand on these findings, reporting age-adjusted and gender-adjusted absolute risk of death/ICU admission of individual biomarkers in a larger, European cohort. In the present study inflammatory markers such as CRP, leucocytes and procalcitonin, and markers of organ damage including eGFR, urea, troponins and D-dimer were associated with higher risk of death/ICU admission within 30 days. However, the association between ferritin and ALAT was non-significant. The association with inflammatory markers and acute phase reactants likely reflects the cytokine storm associated with severe infection and subsequent end-organ damage from severe sepsis. ${ }^{17}$ However, it is also essential to recognise that the acute phase reactants are non-specific markers of inflammation. For example, procalcitonin secretion is not induced by gamma-interferon (produced mainly in response to viral infections), making it primarily an attractive marker of bacterial infections. ${ }^{18}$ Nevertheless, bacterial superinfection is an important consideration in COVID-19; for example, in the study by Wang et al, $81.7 \%$ of patients who died with COVID-19 had an associated bacterial infection. ${ }^{19}$ Probably, patients with longer and more complex admissions or ICU treatment are more vulnerable to secondary infections for example, ventilatoracquired infection, with an expected rise in procalcitonin levels. Accordingly, Zhou et al observed that half of the non-survivors experienced a secondary infection, with almost one-third due to ventilator-associated pneumonia. ${ }^{5}$ However, the association between higher absolute risk of death/ICU admission and elevated baseline procalcitonin in the present study suggests that early concomitant bacterial infection (not only secondary infection) is a significant factor in adverse prognosis for patients with COVID-19 that clinicians should be aware of.

In the study including 191 patients from Wuhan region, leucocytes, older age, higher SOFA score and a higher D-dimer (greater than $1 \mu \mathrm{g} / \mathrm{L}$ on admission) were all associated with in-hospital mortality in patients with COVID-19. ${ }^{5}$ Although the SOFA score is mainly a diagnostic marker of sepsis and septic shock, it may reflect the state and degree of multiorgan dysfunction due to infection in general. ${ }^{20}$ In the present study, abnormal values of biomarkers reflecting organ damage including troponin, eGFR, urea and D-dimer were all associated with higher absolute risk of 30-day mortality and ICU admission. Myocarditis is a known morbidity among patients with COVID-19. ${ }^{21}$ Elevated troponin may reflect myocardial injury among patients with COVID-19, including direct damage to the cardiomyocytes, systemic inflammation, myocardial interstitial fibrosis, coronary plaque destabilisation and hypoxia. ${ }^{21}$ Furthermore, patients with cardiovascular disease are more likely to be admitted with COVID-19, with greater risk of cardiac involvement as their symptoms develop. ${ }^{22}$ With respect to the abnormalities observed in D-dimer, research suggests that respiratory failure in COVID-19 is not only related to development of the acute respiratory distress syndrome, but also due to microvascular thrombotic processes, which are associated with elevated D-dimer. ${ }^{23}$ Finally, beyond the high mortality observed in those with organ dysfunction and respiratory failure, a generalised coagulopathy has also been noted in those with poor prognosis. $^{24}$

\section{Strengths and limitations}

The main strength of this study is the completeness of data from a nationwide, European cohort and the avoidance of selection bias resulting from race, age, sex, socioeconomic status, affiliation to selected hospitals or healthcare systems. The Danish National Patient Registry and the Danish Registry of Medicinal Product Statistics are known to be accurate. ${ }^{25}{ }^{26}$ The main limitation of our study is its observational non-randomised design. There is a lack of information about important clinical parameters, including blood pressure, body mass index and smoking habits.

This study included only patients admitted to the hospital with COVID-19 and with measured baseline biochemical data. Therefore, it is likely to represent symptomatic patients, who are more likely to be elderly or with more comorbidities and at the more severe end of the disease spectrum. Furthermore, patients in the cohort may present to hospital at differing stages of their disease. A large proportion of patients with confirmed COVID-19 in Denmark had missing biochemistry data, which most likely represent patients who attended the emergency room with mild symptoms, which did not warrant admission or blood tests, and were not included in this study, thus leading to selection bias and limiting generalisability. Some biomarkers (particularly D-dimer, troponin and procalcitonin) are likely to be measured in those with the most severe disease (confounding by indication). 


\section{CONCLUSIONS}

In this nationwide study of patients admitted with COVID19, elevated levels of CRP, leucocytes, procalcitonin, urea, troponins and D-dimer, and low levels of eGFR were associated with higher standardised absolute risk of death/ ICU admission within 30 days.

\section{Author affiliations}

${ }^{1}$ Department of Cardiology, Copenhagen University Hospital Herlev and Gentofte, Hellerup, Denmark

${ }^{2}$ Department of Cardiology, Zealand University Hospital, Roskilde, Denmark ${ }^{3}$ The Danish Heart Foundation, Department of Research, Copenhagen, Denmark ${ }^{4}$ William Harvey Research Institute, Barts and the London School of Medicine and Dentistry, Queen Mary University of London, London, UK

${ }^{5}$ Unit of Clinical Biostatistics and Epidemiology and Department of Cardiology, Aalborg University Hospital, Aalborg, North Denmark Region, Denmark

${ }^{6}$ Department of Cardiology, North Denmark Regional Hospital \& Aalborg University Hospital, Aalborg, Denmark

${ }^{7}$ Department of Cardiology, Rigshospitalet, University of Copenhagen, Copenhagen, Denmark

${ }^{8}$ The National Institute of Public Health, University of Southern Denmark, Copenhagen, Denmark

${ }^{9}$ Department of Clinical Investigation and Cardiology, North Zealand Hospital, Hillerød, Denmark

${ }^{10}$ Department of Cardiology, Bispebjerg-Frederiksberg Hospital, University of Copenhagen, Copenhagen, Denmark

Contributors Authors GH, JP, A-MSO, PM, MA, MK, KK, LK, GHG, CT-P and CNB made substantial contributions to the conception and design of the work. Author $\mathrm{JP}$ was primarily responsible for the acquisition and analysis, and authors CNB, GH and JP were primarily responsible for interpretation of data for the work. All authors were involved in the drafting, revision and final approval of the published version, and agree to be accountable for all aspects of the work.

Funding The authors have not declared a specific grant for this research from any funding agency in the public, commercial or not-for-profit sectors.

Competing interests None declared.

Patient consent for publication Not required.

Ethics approval The Data Responsible Institute (The Capital Region of Denmark) approved this study (ref. no.: P-2019-191), in line with the General Data Protection Regulation (GDPR) and The Data Protection Act.

Provenance and peer review Not commissioned; externally peer reviewed.

Data availability statement No data are available. Due to the nature of this research, participants of this study did not agree for their data to be shared publicly, so supporting data are not available.

Supplemental material This content has been supplied by the author(s). It has not been vetted by BMJ Publishing Group Limited (BMJ) and may not have been peer-reviewed. Any opinions or recommendations discussed are solely those of the author(s) and are not endorsed by BMJ. BMJ disclaims all liability and responsibility arising from any reliance placed on the content. Where the content includes any translated material, BMJ does not warrant the accuracy and reliability of the translations (including but not limited to local regulations, clinical guidelines, terminology, drug names and drug dosages), and is not responsible for any error and/or omissions arising from translation and adaptation or otherwise.

Open access This is an open access article distributed in accordance with the Creative Commons Attribution Non Commercial (CC BY-NC 4.0) license, which permits others to distribute, remix, adapt, build upon this work non-commercially, and license their derivative works on different terms, provided the original work is properly cited, appropriate credit is given, any changes made indicated, and the use is non-commercial. See: http://creativecommons.org/licenses/by-nc/4.0/.

\section{ORCID iD}

Gethin Hodges http://orcid.org/0000-0002-2338-6722

\section{REFERENCES}

1 World Health Organization. Coronavirus disease (COVID-19) outbreak. Available: https:// www.who.int

2 Guan W-J, Ni Z-Y, Hu Y, et al. Clinical characteristics of coronavirus disease 2019 in China. N Engl J Med 2020;382:1708-20. doi:10.1056/NEJMoa2002032

3 Yang X. Articles clinical course and outcomes of critically ill patients with SARS-CoV-2 pneumonia in Wuhan, China: a single-centered, retrospective, observational study. Lancet 2020;8:P475-81.

4 Inciardi RM, Lupi L, Zaccone G, et al. Cardiac involvement in a patient with coronavirus disease 2019 (COVID-19). JAMA Cardiol 2020;5:819.

5 Zhou F, Yu T, Du R, et al. Clinical course and risk factors for mortality of adult inpatients with COVID-19 in Wuhan, China: a retrospective cohort study. Lancet 2020;395:1054-62. doi:10.1016/S01406736(20)30566-3

6 Wynants L, Van Calster B, Collins GS, et al. Prediction models for diagnosis and prognosis of covid-19 infection: systematic review and critical appraisal. BMJ 2020;369:m1328.

7 Truog RD, Mitchell C, Daley GQ. The Toughest triage - allocating ventilators in a pandemic. N Engl J Med 2020;382:1973-5.

8 Huang C. Clinical features of patients infected with 2019 novel coronavirus in Wuhan, China, 2020. Available: www.thelancet.com

9 Chen N, Zhou M, Dong X, et al. Epidemiological and clinical characteristics of 99 cases of 2019 novel coronavirus pneumonia in Wuhan, China: a descriptive study. Lancet 2020;395:507-13.

10 Lippi G, Lavie CJ, Sanchis-Gomar F. Cardiac troponin I in patients with coronavirus disease 2019 (COVID-19): evidence from a metaanalysis. Prog Cardiovasc Dis 2020;63:390-1.

11 Kermali M, Khalsa RK, Pillai K, et al. The role of biomarkers in diagnosis of COVID-19 - A systematic review. Life Sci 2020;254:117788.

12 Andersen TF, Madsen M, Jørgensen J, et al. The Danish national Hospital register. A valuable source of data for modern health sciences. Dan Med Bull 1999;46:263-8.

13 Beigel JH, Tomashek KM, Dodd LE, et al. Remdesivir for the treatment of Covid-19 - Preliminary report. N Engl J Med 2020;383:993-4. doi:10.1056/NEJMoa2007764

14 Martins-Filho PR, Tavares CSS, Santos VS. Factors associated with mortality in patients with COVID-19. A quantitative evidence synthesis of clinical and laboratory data. Eur J Intern Med 2020;76:97-9.

15 Cecconi M, Piovani D, Brunetta E, et al. Early predictors of clinical deterioration in a cohort of 239 patients hospitalized for Covid-19 infection in Lombardy, Italy. J Clin Med 2020;9:1548.

16 Henry BM, de Oliveira MHS, Benoit S, et al. Hematologic, biochemical and immune biomarker abnormalities associated with severe illness and mortality in coronavirus disease 2019 (COVID-19): a meta-analysis. Clin Chem Lab Med 2020;58:1021-8.

17 Mehta P, McAuley DF, Brown M, et al. COVID-19: consider cytokine storm syndromes and immunosuppression. Lancet 2020;395:1033-4.

18 Samsudin I, Vasikaran SD. Clinical utility and measurement of procalcitonin. Clin Biochem Rev 2017;38:59-68.

19 Wang L, He W, Yu X, et al. Coronavirus disease 2019 in elderly patients: characteristics and prognostic factors based on 4-week follow-up. J Infect 2020;80:639-45.

20 Singer M, Deutschman CS, Seymour CW, et al. The third International consensus definitions for sepsis and septic shock (sepsis-3). JAMA 2016;315:801-10.

21 Babapoor-Farrokhran S, Gill D, Walker J, et al. Myocardial injury and COVID-19: possible mechanisms. Life Sci 2020;253:117723.

22 Madjid M, Safavi-Naeini P, Solomon SD, et al. Potential effects of coronaviruses on the cardiovascular system: a review. JAMA Cardiol 2020;5:831.

23 Magro C, Mulvey JJ, Berlin D, et al. Complement associated microvascular injury and thrombosis in the pathogenesis of severe COVID-19 infection: a report of five cases. Trans/ Res 2020;220:1-13.

24 Tang N, Li D, Wang X, et al. Abnormal coagulation parameters are associated with poor prognosis in patients with novel coronavirus pneumonia. J Thromb Haemost 2020;18:844-7.

25 Schmidt M, Schmidt SAJ, Sandegaard JL, et al. The Danish national patient registry: a review of content, data quality, and research potential. Clin Epidemiol 2015;7:449.

26 Schmidt M, Pedersen L, Sørensen HT. The Danish civil registration system as a tool in epidemiology. Eur J Epidemiol 2014;29:541-9. 